\title{
An interactive optical tweezers simulation for science education
}

\author{
Thomas T. Perkins $^{*}{ }^{*}$, Christopher V. Malley ${ }^{\mathrm{c}}$, Michael Dubson ${ }^{\mathrm{d}}$, Katherine K. Perkins ${ }^{\mathrm{d}}$ \\ a JILA, National Institute of Standards and Technology and University of Colorado, Boulder, CO, \\ USA 80309 \\ ${ }^{b}$ Dept. of Molecular, Cellular, and Developmental Biology, University of Colorado, Boulder, CO, \\ USA 80309 \\ ${ }^{\mathrm{c}}$ PixelZoom, Inc., Boulder, CO, USA 80309 \\ ${ }^{\mathrm{d} D e p t}$. of Physics, University of Colorado, Boulder, CO, USA 80309
}

\begin{abstract}
We developed a freely available interactive simulation of optical traps and their biological applications (phet.colorado.edu). The target audience is undergraduate majors as well as more advanced researchers. The simulation has three panels: optical traps, manipulating DNA, and measuring molecular motors. Each panel has options that allow students to interactively explore key physical ideas. For instance, viscosity can be turned off to see the critical aspect of dissipation, or time can be slowed down to see the oscillating electric field and the induced charge separation. An overview of the simulation and specific exercises suitable for an undergraduate class are discussed.
\end{abstract}

Keywords: optical tweezers, optical trap, single molecule, biophysics, teaching, education

\section{INTRODUCTION}

Educational simulations (sims) can be highly engaging and effective tools for helping students learn science and math. ${ }^{1}$ However, they must be carefully developed and tested. Since 2002, the PhET Interactive Simulations project at the University of Colorado has been working to provide these powerful learning tools to students and teachers worldwide. The project has developed over 85 interactive simulations for teaching and learning science, all of which are available for free from the PhET website (http://phet.colorado.edu). Simulations like Energy Skate Park, Circuit Construction Kit, and Gas Properties create animated, interactive, game-like environments in which students learn through scientist-like exploration. The sims emphasize the connections between real-life phenomena and the underlying science. They make the invisible visible (e.g. electrons, photons, field vectors), include the visual models that experts use to aid their thinking, animate those models, and provide interactive controls and measuring tools - design features which help students build their own understanding and skills.

What's special about a PhET sim? While there are many science sims available on the web, the PhET project was started by Nobel Laureate Carl Wieman specifically to improve the way that science is taught and learned by creating sims that are aligned with and vetted through educational research. Each sim is developed by a team of scientists, software engineers, science educators, and science education researchers to facilitate learning, while often addressing known student difficulties with the content. The PhET project is actively engaged in research on effective design and classroom use of interactive simulations. As a result of this process, every sim is tested through student interviews and observations of use. Although PhET sims can be used in a variety of ways, they are specifically designed to make scientist-like, inquiry-based activities productive and fun learning experiences for students.

Optical tweezers are revolutionizing biophysics. At the interface between biology and physics, they provide an excellent opportunity to educate students about a diverse set of key physical principles in biophysics, including Brownian motion, equipartition theorem, and dissipation. Using the sim presented here, students can manipulate single DNA molecules and measure the motion of molecular motors.

*tperkins@jila.colorado.edu; phone: 1303 492-5291; fax: 1303 492-5235

Optical Trapping and Optical Micromanipulation VII, edited by Kishan Dholakia, Gabriel C. Spalding, Proc. of SPIE Vol. 7762, 776215 - (c) 2010 SPIE · CCC code: 0277-786X/10/\$18 · doi: 10.1117/12.862030 


\section{OVERVIEW OF OPTICAL TWEEZERS SIMULATION}

The sim is divided into three main panels that emphasize the physics of optical tweezers (Fig. 1), manipulating DNA with optical tweezers (Fig. 2), and measuring molecular motors (Fig. 3). We designed the sim to present an appealing environment that literally invites the student to interact and explore in an open-style play area. All the controls are simple and intuitive, e.g. click-and-drag manipulation, sliders, and radio buttons. For instance, the trapping laser can be moved by manipulating the focusing lens or laser assembly. Handles on the laser as well as changes in the mouse icon provide visual cues to the student. The mouse icon also changes when hovering the mouse over the bead, inviting the student to drag the bead to new areas on the screen and observing the results. Each main panel will be briefly discussed. Examples of student activities associated with these panels will be discussed in $\S 3$.

\subsection{Physics of Tweezers}

This panel has numerous options allowing the student to explore a wide range of activities, including turning off Brownian motion and/or varying dissipation (including trapping a bead in vacuum). It is quite fun to grab the laser and pull it rapidly to the side and then slowly move it back towards the bead and watch it get trapped. Beyond such exploratory activities, more serious learning goals can be established. For instance, how does optical trapping work? One explanation starts with the induced dipole moment in a neutral bead. Figure 1 allows students to directly visualize this induced dipole. They can even turn on the "show optical trap force" and see the force oscillate with the electric field. For more advanced students, you can ask why you have to use focused light to generate a local, three dimensional maximum in the electric field?

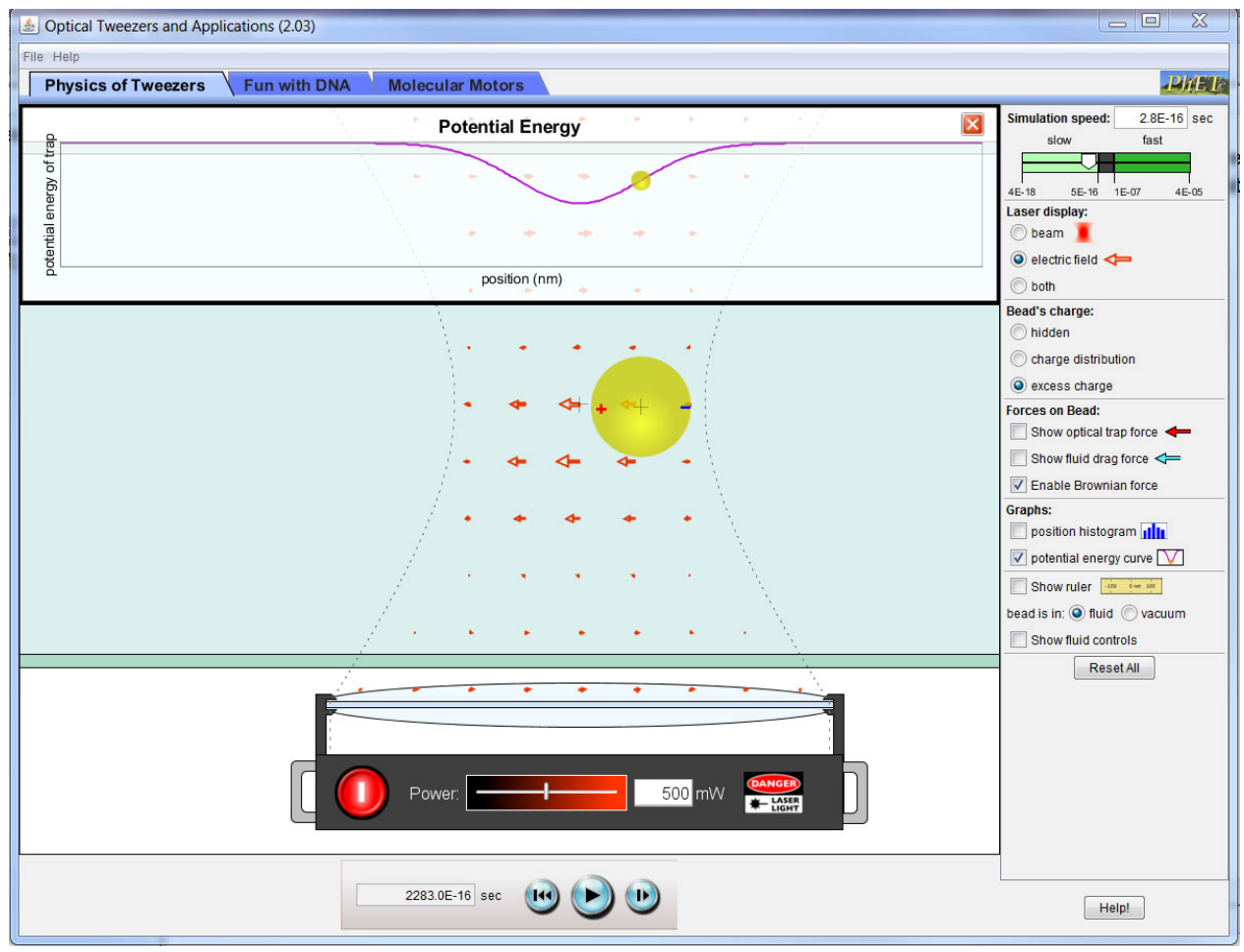

Fig. 1. Physics of Tweezers tab. In this screen shot, the activity is to visualize the induced charge on the bead caused by the oscillating electric field of the laser. Since the oscillation frequency of lasers is very fast, the time step of the sim is dramatically slowed down (upper right corner). Visualizing the induced dipole moment and the local electric field are enabled by selecting the corresponding check boxes in the control area (excess charge and electric field, respectively). The average potential energy curve is also shown with the bead at an offset from the trap center for clarity. Note: No significant motion of the bead occurs on this time scale as is physically accurate. To see such motion, the simulation speed slider needs to be in the fast region (dark green). 


\subsection{Fun with DNA}

The ability to manipulate and measure the motion of biological molecules is one of optical tweezers' most important applications. DNA, one of biology's best known molecules, is often used in optical tweezers experiments. One concept in biophysics that students have difficulty understanding is entropic elasticity. In other words, the elasticity of DNA (and polymers in general) does not arise from stretching chemical bonds but aligning the polymers and reducing their entropy. This concept is often discussed in the context of the random walks and the number of paths connecting two points, very similar to what is illustrated in Figure 2, where a DNA molecule is stretched. The forces of both the trap and the DNA are shown. Moreover, the sim shows the rapidly fluctuating configurations of the DNA that are crucial for a polymer to explore its accessible states. Thus, this sim lets students explicitly see the reduced entropy. To more directly connect this change in entropy to the real world, a simple demonstration is valuable. Have the students place a latex glove on their hand and rapidly stretch and relax it. They should feel the heating and cooling on the backs of their hand, giving them a macroscopic method to understand the flow of heat into and out of a polymer as it is stretched and relaxed. Thus, the sim, in combination with a simple lab demonstration, can be a valuable tool for illustrating a difficult to grasp concept.

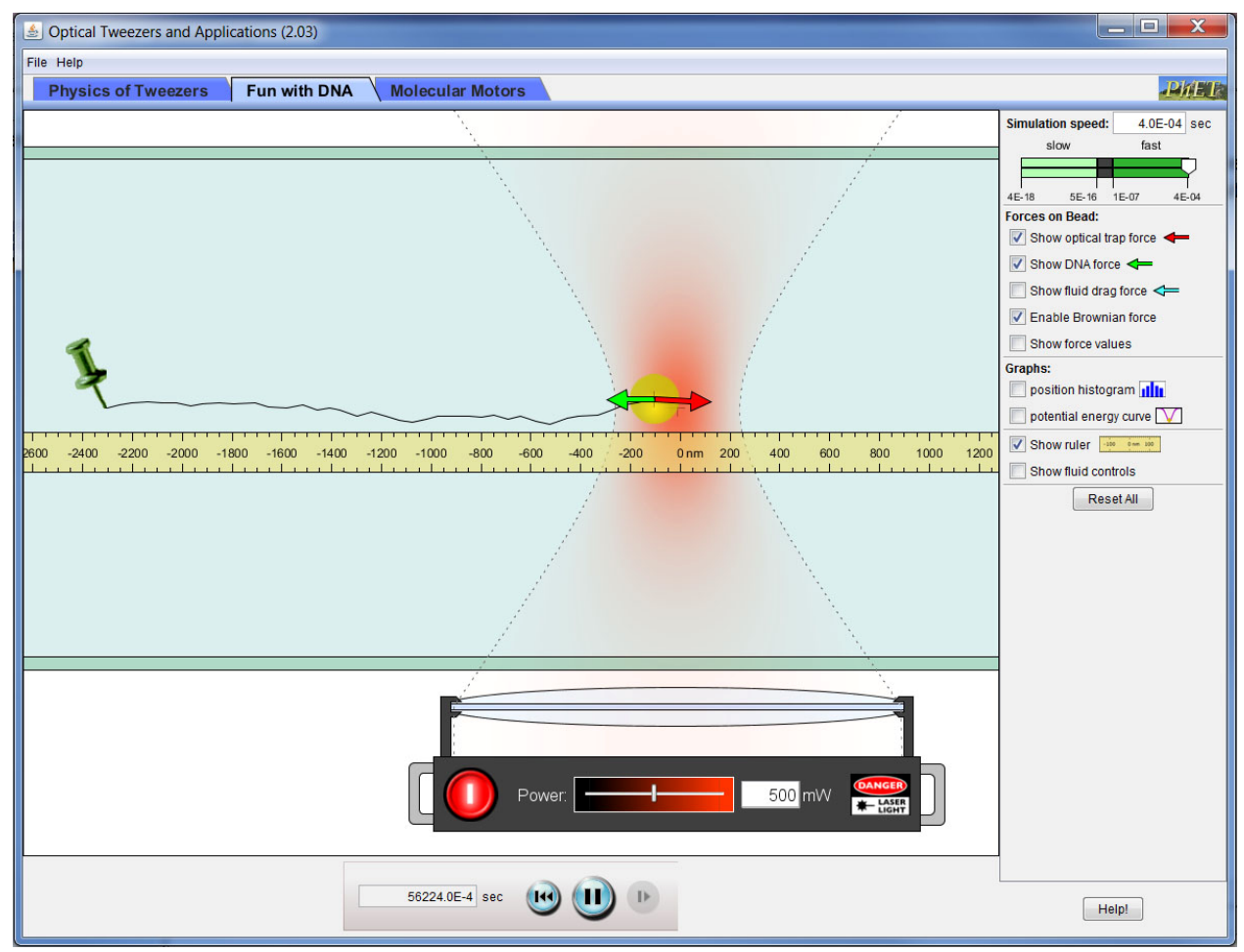

Fig. 2. Fun with DNA tab. In this screen shot, the DNA is stretched taut with the optical tweezers. The sim shows both the DNA strand and the bead undergoing vigorous Brownian motion. The two primary forces acting on the bead besides Brownian motion are shown: the force from stretching the DNA (green) and the trapping force from the optical tweezers $(r e d)$. The distance from the center of the optical trap can be measured by selecting the "show ruler" box.

\subsection{Molecular Motors}

Enzymes that move are called molecular motors. Optical-trapping experiments have yielded a wealth of information about translocation rates and step sizes of various molecular motors. For simplicity, the sim focuses on proteins that move along DNA. Optical tweezers allow controlled loads (e.g. force) to be applied, allowing the motor's velocity (e.g., enzymatic rate) to be measured as a function of applied load. Such experiments yield insight into the mechanisms of these molecular motors. 
The primary goal of this panel is to allow students to mimic measuring molecular motors. If the laser power is low enough, they can watch the enzyme exert enough force to pull the bead out of the optical trap. Or, at high force (power set to $1000 \mathrm{~mW}$ ), they can watch the trap essentially stall the enzyme. Enzymatic rate is most simply seen by a small white line rotating like the hand on a clock. Students can also simulate more sophisticated experiments using a force clamp, which automatically moves the trap so the force is held constant - a common method to study molecular motors.

The sim also allows for the student to investigate alternative ways to measure molecular motors. Figure 3 demonstrates watching a molecular motor move the bead against a force provided by a fluid flow. Forces provided by such fluid flows were instrumental in the first measurement of DNA elasticity, ${ }^{2}$ and continue to be popular in the study of complex molecular motors moving along DNA. An excellent question for students is: why use such a lower resolution technique now, given the sophistication of modern optical tweezers? In part, the answer is the simplicity of establishing the biological assay and the ability to do numerous single-molecule experiments in parallel.

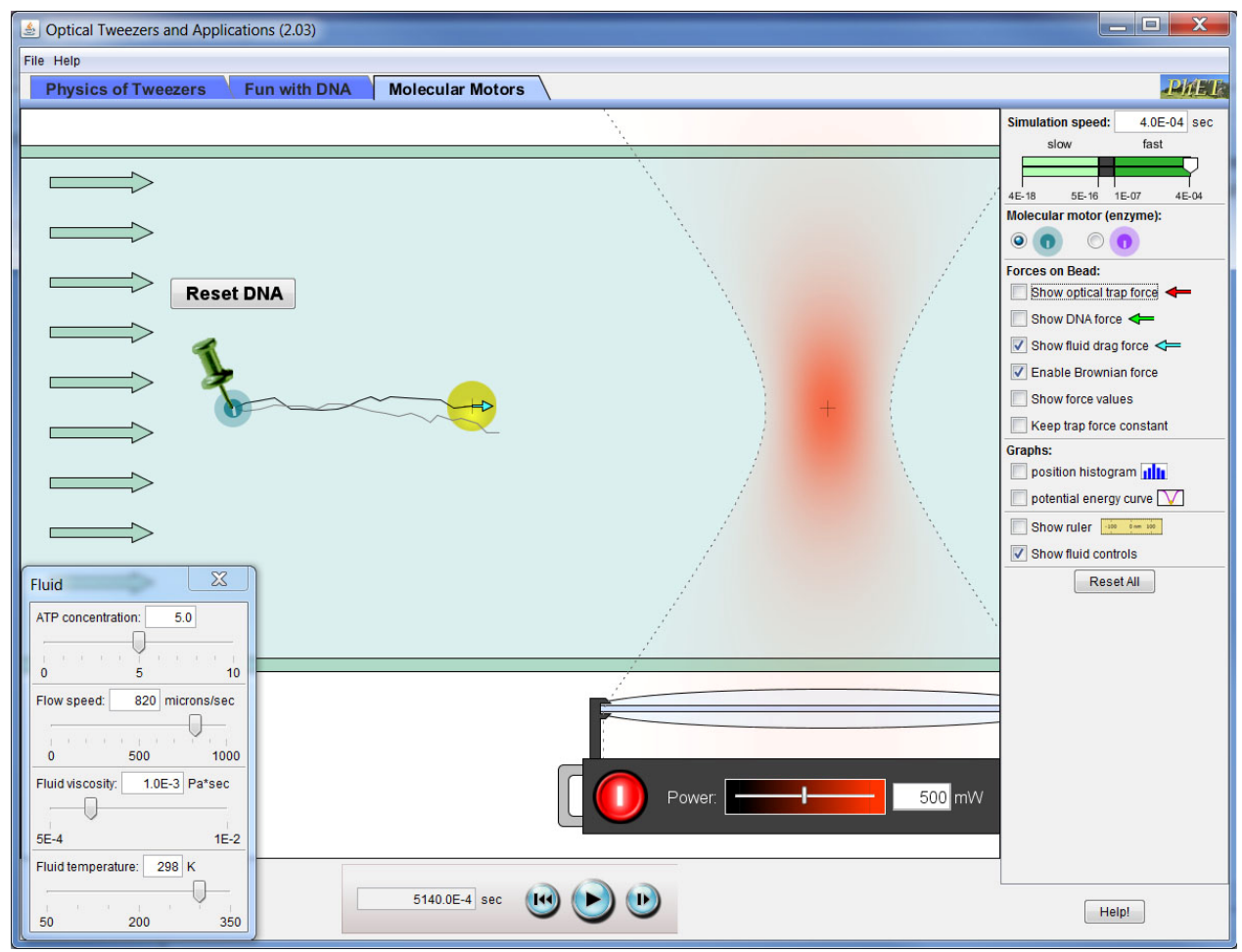

Fig. 3. Molecular motors tab. In this screen shot, the optical tweezers are moved to the far right of the screen so the bead is not optically trapped. The main learning goal in this screen shot is for the student to realize that simple fluid flows provide an alternative means to stretch DNA and thereby quantify enzymatic motion. In the absence of flow or optically trapping the bead, the DNA would entropically relax to a random coil. To prevent this relaxation, the DNA is stretched by hydrodynamic flow. The fluid flow affects the input (black) and output (grey) strand as well as the bead (yellow). To control the flow, the "show fluid controls" box is checked. The magnitude and the viscosity of the fluid flow can be changed. This panel is also used control the ATP concentration which affects the velocity of the enzyme. As shown, the ATP concentration is relatively high, leading to fast motion. The sim shows the enzyme pulling in the DNA. The two different molecular motors (turquoise and purple circles) have different force velocity characteristics. Advanced students can quantify this difference, with the simplest method to optically trap the bead and then enable a force clamp ("keep trap force constant" box).

\section{ACTIVITIES}

Students can engage in a number of qualitative and quantitative activities within the sim to emphasize central aspects of optical tweezers. 


\subsection{Measuring trap stiffness}

The complexity in optical tweezers for single-molecule biophysics lies in making calibrated measurements of biochemically active systems. Calibrated measurements allow the detailed study of kinetics ${ }^{3}$ and energetics ${ }^{4}$ of biological systems. Figure 4 demonstrates making quantitative measurements using the sim to calibrate the optical tweezers.

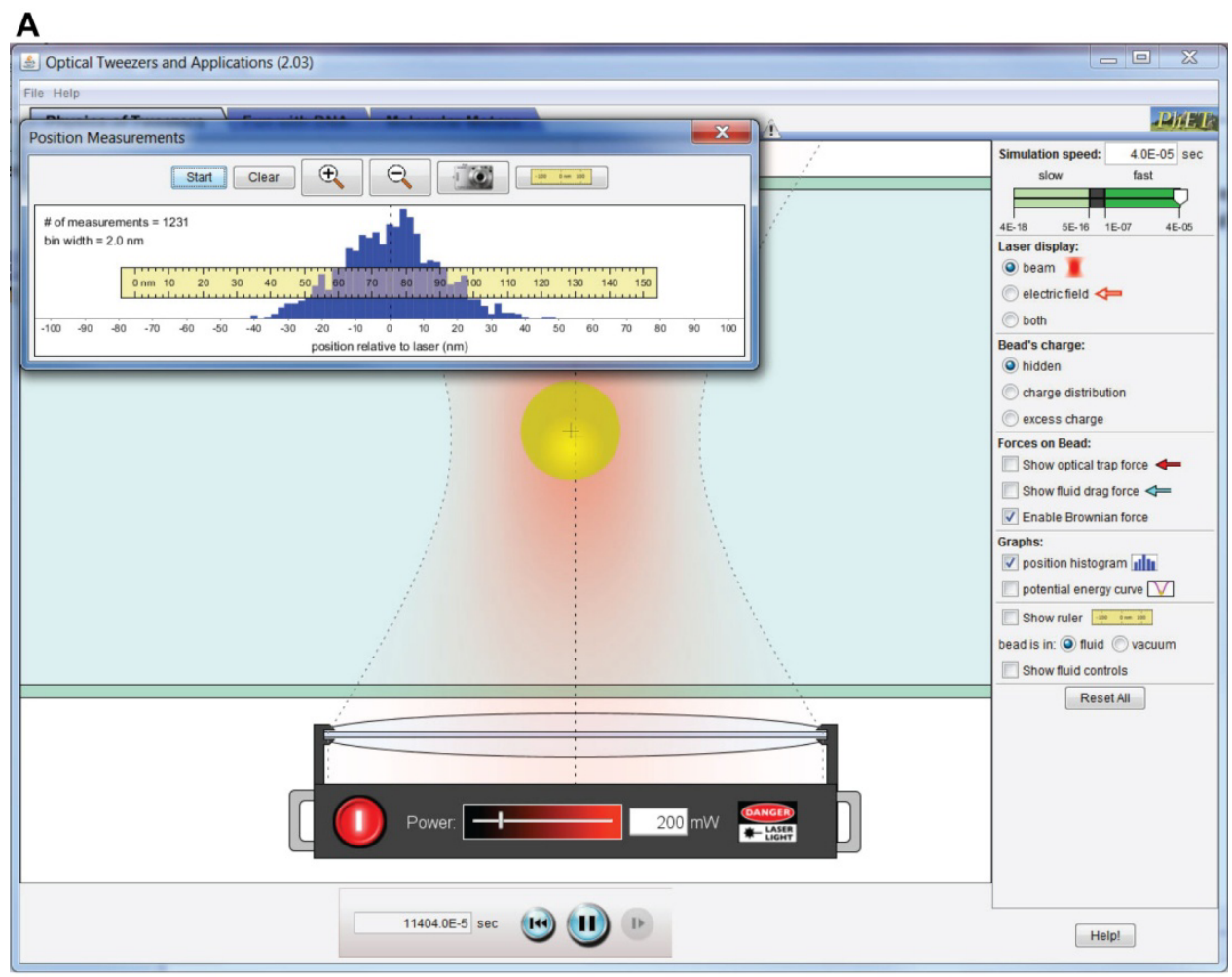

B
\begin{tabular}{|r|c|c|c|}
\hline Power & FWMH & $\sigma_{\text {bd }}$ & $\mathrm{k}_{\text {trap }}$ \\
\hline 200 & 30 & 12.5 & 0.026 \\
\hline 400 & 20 & 8.33 & 0.059 \\
\hline 600 & 16 & 6.67 & 0.092 \\
\hline 800 & 15 & 6.25 & 0.105 \\
\hline 1000 & 12 & 5 & 0.164 \\
\hline
\end{tabular}

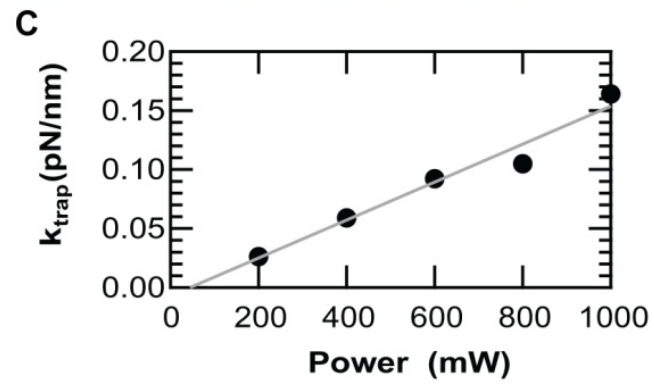

Fig. 4. Making quantitative measurements. (a) In this screen capture, the main goal is to calibrate the stiffness $\left(k_{\text {trap }}\right)$ of the tweezers. To do so, the laser power is set and the resulting Brownian motion measured. Measurements are made by selecting the position histogram and waiting for the histogram to be populated. More points lead to more accurate measurements. The "+" and "-" button scale the ruler to optimize it to the width of the data. The "camera" icon takes a snap shot and saves the measurement in a new window. The affect of laser power on $k_{\text {trap }}$ is investigated by increasing the power followed by clicking the "clear" button to reset the histogram. (b) Measurements of the full width half max (FWHM) based of the histogram of bead motion at different laser powers. To calculate $k_{\text {trap }}$, the equipartioin theorem is used which requires the standard deviaition in the data $\left(\sigma_{\mathrm{bd}}=\mathrm{FWHM} / 2.4\right)$. (c) $k_{\text {trap }}$ as a function of laser power. Note the fact that the line does not intersect 0 for this data is a good talking point about the accuracy of the data and the need to do multiple trials per data point.

\subsection{Additional suggested activities}

The sim leads to a wide range of activities that are too broad to fully document within this report. Thus, we briefly outline a number of other activities that seek to emphasize certain learning goals of the optical tweezers sim. 
Dissipation is a central concept in optical tweezers (and cooling of atoms in optical traps for atomic physics). Without such dissipation, the bead will oscillate back and forth. The learning goal is to emphasize the necessity of dissipation for trapping the bead at the bottom of the optical tweezers. The activity is to have the students click on the bead and pull it to the left, and then release it. Have the students neatly sketch the resulting motion with the simulation set to water and to vacuum. As an added activity, the students can turn of the Brownian motion and repeat the process.

Optical traps can be thought of as potential wells. The learning goal is for the student to visualize the change in the average Brownian motion with increasing stiffness (and depth) of the the optical trap. The simple activity is for the student to sketch the potential energy curves at four different laser powers $(200,400,800$, and $1000 \mathrm{~mW})$. Finally, the student can set the laser power to a minimum power $(5 \mathrm{~mW})$ and record the time it takes for the bead to leave the optical tweezers.

In $\S 3.1$, the stiffness of the optical tweezers is established. However, hydrodynamic drag represents the best way to establish the linearity $\left(F=-k_{\text {trap }} x_{\mathrm{bd}}\right)$ of an optical tweezers where $x_{\mathrm{bd}}$ is the displacement of the bead from the center of the optical tweezers. The learning goal is for the students to apply Stokes drag to demonstrate the linearity of the optical trap. The activity is for the students to establish a fluid flow, measure resulting displacement using the ruler at different velocities and then plot the resulting data on a graph. Stokes drag is given by $\left(F_{\text {drag }}=6 \pi \eta r v_{\text {fluid }}\right)$ where $\eta$ is the viscosity of the fluid and $r_{\mathrm{bd}}$ is the radius of the trapped bead. For advanced students, they can combine this activity with the calibration of the trap stiffness $(\S 3.1)$ to determine the bead radius $\left(r_{\mathrm{bd}}=160 \mathrm{~nm}\right)$.

\section{CONCLUSIONS}

Biophysics classes are increasingly common in undergraduate physics curriculums, and this sim provides a means for undergraduates to interact with a cutting edge research tool. We expect this sim to be useful for a range of students, from undergraduates to graduate students. More generally, scientists new to physical measurements of biological systems can interactively explore the fundamental physical principles of how optical tweezers work and methods to measure molecular motors.

\section{Acknowledgments}

This work was supported by the National Science Foundation (Phy-0404286, Phy-0551010), the William and Flora Hewlett Foundation, and NIST. Mention of commercial products is for information only; it does not imply NIST's recommendation or endorsement, nor does it imply that the products mentioned are necessarily the best available for the purpose. TTP is a staff member of NIST's Quantum Physics Division.

\section{REFERENCES}

[1] Wieman, C. E., Adams, W. K. and Perkins, K. K., "PHYSICS: PhET: simulations that enhance learning," Science 322(5902), 682-683 (2008).

[2] Smith, S. B., Finzi, L. and Bustamante, C., "Direct Mechanical Measurements of the Elasticity of Single DNA Molecules by Using Magnetic Beads," Science 258, 1122-1126 (1992).

[3] Svoboda, K. and Block, S. M., "Force and velocity measured for single kinesin molecules," Cell 77(5), 773-784 (1994).

[4] Yin, H., Wang, M. D., Svoboda, K., Landick, R., Block, S. M. and Gelles, J., "Transcription Against an Applied Force," Science 270, 1653-1657 (1995). 